\title{
Nach der Ad-hoc-Digitalisierung: Was bleibt? Was soll weg?
}

\author{
Jana Riedel, Anja Scbulz und Julia Henschler
}

\section{Zusammenfassung des Beitrags}

Der Beitrag trägt die Ergebnisse aus drei Think Tanks zusammen, die nach einem Jahr des digitalen Lehrbetriebs an sächsischen Hochschulen mit unterschiedlichen Akteursgruppen online durchgeführt wurden. Im Rahmen der Veranstaltungen erfolgte eine Reflexion der aus einer Notsituation erreichten Fortschritte im Bereich der digitalen Hochschullehre. Die Ergebnisse wurden in Form von Thesen aufbereitet und in den öffentlichen Diskurs eingebracht. Im vorliegenden Beitrag werden die entstandenen Thesen und Argumentationslinien samt ihrer landesspezifischen Besonderheiten diskutiert.

Schlüsselbegriffe: Hochschullebre $\bullet$ Post-Corona $\bullet$ digital gestützte Lehre $\bullet$ digitale Hochschulbildung $\bullet$ Think Tank

\section{Ausgangslage: Lehre im Notbetrieb an sächsischen Hochschulen \\ Zustand der digitalen Lebre an sächsischen Hochschulen im Pandemiebetrieb}

Die sächsischen Hochschulen befinden sich seit dem Frühjahr 2020 im Notbetrieb. Während im Sommersemester 2020 eine Pflicht zur digital gestützten Durchführung aller Lehrveranstaltungen bestand, wurden in den Folgesemestern (Wintersemester (WiSe) 2020/21, Sommersemester (SoSe) 2021) Ausnahmen zugelassen, bspw. für Laborpraktika, für die ein Arbeiten vor Ort unerlässlich ist, oder für ausgewählte Erstsemesterveranstaltungen, um den Einstieg ins Studium zu erleichtern. Da der über das Deutsche Forschungsnetz bereitgestellte Videokonferenzdienst Adobe Connect nur bedingt verfügbar war, schafften sich die Hochschulen jeweils eigene Lizenzen für Videokonferenzdienste an. Für kleinere Veranstaltungen wurden Instanzen von BigBlueButton eingerichtet und wegen ihrer Installation auf hochschuleigenen Servern und der daraus entstehenden Möglichkeit zur Einhaltung des Datenschutzes empfohlen, für größere Veranstaltungen wurden Lizenzen für GoToMeeting und Zoom beschafft. Der Prüfungsbetrieb wurde an den einzelnen Hochschulen unterschiedlich stark digitalisiert. So schafften einige Hochschulen Möglichkeiten für die Durchführung von Präsenzprüfungen, andere führten eigene Prüfungsinstanzen für digitale Klausuren ein. Das Format der Open-Book-Klausuren wurde als präferierte Lösung unterstützt, außerdem gab es 
an nahezu allen Hochschulen eine erweiterte Freiversuchsregelung, die es den Studierenden erlaubt, Klausurergebnisse zu annullieren, ohne dass diese Teilnahme als eigener Versuch gewertet wird.

Die in Sachsen etablierte Routine einer hochschulübergreifenden Nutzung und Weiterentwicklung der technischen Infrastruktur (z. B. Lernmanagementsystem (LMS) OPAL ${ }^{1}$, Videocampus Sachsen) durch einen zentralen Dienstleister wurde für den Einsatz der Videokonferenzdienste und Prüfungsinstanzen um hochschulspezifische Serverinstallationen oder Lizenzen ergänzt. Die Nutzungszahlen und die Anzahl der verfügbaren Ressourcen in den in Sachsen zentral bereitgestellten Lerninfrastrukturen sind in den digital durchgeführten Semestern (SoSe 2020, WiSe 2020/21) deutlich gestiegen (vgl. AK E-Learning (in press)). So hat sich die Nutzungsintensität (Anzahl Kursaufrufe je Nutzende*r im Semester) des LMS OPAL, die Anzahl der Aufrufe von Testressourcen in der Lernplattform sowie die Anzahl verfügbarer Medien in der Videoplattform gegenüber dem Vorjahressemester jeweils verdoppelt (vgl. ebd.). In den Vorjahren war hingegen eine Stagnierung der Nutzungsintensitäten zu beobachten (vgl. ebd.), wie auch andere Erhebungen im bundesdeutschen Raum zeigen (vgl. Zawacki-Richter 2020). Dennoch war die Nutzung der Lernplattform nicht flächendeckend, denn nur etwa 7 von 10 immatrikulierten Studierenden haben OPAL aktiv genutzt ${ }^{2}$ (vgl. ebd.).

Laut einer Befragung unter sächsischen Studierenden ist es den Hochschulen gelungen, den Lehrbetrieb im Sommersemester 2020 auch ohne Präsenzveranstaltungen aufrechtzuerhalten, sodass die Fortführung des Studiums grundsätzlich möglich war (vgl. Karapanos et al. 2021). Die Mehrheit der Studierenden hatte hierbei eine ausreichende Ausstattung für den Zugriff auf digitale Materialien, wobei fast alle (96\%) Studierenden über ein eigenes internetfähiges Endgerät, jedoch nur etwa drei Viertel über eine stabile Internetverbindung verfügten. Darüber hinaus hat jeweils ca. ein Siebtel der Studierenden einen Mangel im Bereich der Ausstattung mit peripheren Geräten (großer Bildschirm, Webcam, Mikrophon) oder eigener Software (z.B. Dokumentenbearbeitung, Präsentationserstellung) (vgl. ebd.).

Die digital gestützte Lehre fand nach Angaben der Studierenden überwiegend unter Nutzung von LMS, digitalen Texten, digitalen Präsentationstools (z.B. PowerPoint) sowie Chatund Konferenzdiensten statt, Werkzeuge zur Kommunikation und Kollaboration wurden kaum angeboten (vgl. ebd.). Die Dominanz dieser Medienformate konnte auch an anderen deutschen Hochschulen festgestellt werden (vgl. Faßbender et al. 2020). Das Angebot deckt sich mit der Zufriedenheit der Studierenden, welche mit den häufig angebotenen Medienformaten eher zufrieden sind, wobei sie mit Medienformaten, in denen sie selbst aktiv werden sollen (Wikis, Blogs, Soziale Netzwerke) eher unzufrieden sind (vgl. Karapanos et al. 2021). Die Studierenden geben an, dass sie mit den angebotenen Videos und Audios zufrieden sind, diese werden jedoch von den Lehrenden eher selten angeboten (vgl. ebd.). Gleichzeitig zeigen Umfragen unter Lehrenden der HTWK Leipzig, dass gerade Videokonferenzen und Videos

\footnotetext{
${ }^{1}$ Das Lernmanagementsystem OPAL wird nahezu von allen sächsischen Hochschulen genutzt. Eine Hochschule nutzt eine selbstgehostete Moodle-Instanz.

${ }^{2}$ Neben der einen Hochschule nutzen auch einige Fakultäten und Lehrstühle eigene Lernmanagementsysteme oder Bereitstellungsmöglichkeiten (z. B. Cloudspeicher-Lösungen) für ihre Angebote.
} 
die beiden Medienformate sind, die die Mehrheit der Lehrenden auch nach dem erhofften Ende der Pandemiesituation weiterhin nutzen wollen (vgl. Hooffacker o. J.).

In der Wahrnehmung der Studierenden war das Studium im Sommersemester 2020 etwas schwieriger zu bewältigen als im Vorjahr. Dabei haben beim Vergleich der Vor- und Nachteile digitaler Lehre die nachteilig erlebten Faktoren (wie fehlende Interaktion mit Peers, mangelnde Möglichkeiten für Interaktion mit Lehrenden) überwogen (vgl. Karapanos et al. 2021). Sowohl Lehrende als auch Studierende der TU Dresden schätzten die digital gestützte Lehre als aufwendiger ein als die Präsenzlehre, wohingegen die Qualität der Online-Lehre von beiden Gruppen überwiegend als niedriger eingestuft wird (vgl. Frohwieser et al. 2020).

Systematische Auswertungen zum Prüfungsbetrieb unter Pandemiebedingungen an sächsischen Hochschulen sind derzeit noch nicht veröffentlicht. Eine erste Umfrage an der HTWK Leipzig zeigt, dass die bevorzugten digital realisierten Prüfungsformate die Durchführung mündlicher Prüfungen per Videokonferenz sowie die Bearbeitung und digitale Einreichung asynchroner Projektarbeiten, Präsentationen oder Belege waren (vgl. Hooffacker/Gomon o.J.). Diese sind auch für den zukünftigen Einsatz akzeptiert (vgl. ebd.). Digitale Klausuren waren hingegen die am seltensten umgesetzte Prüfungsform und werden auch für die Zukunft nur von wenigen Lehrenden in Betracht gezogen (vgl. ebd.).

\section{Projekthintergrund}

Das sächsische Verbundprojekt Digitalisierung der Hocbscbulbildung in Sacbsen (DHS, 20192023, gefördert durch das Sächsische Staatsministerium für Wissenschaft, Kultur und Tourismus (SMWK)) ist ein gemeinsames Vorhaben des Hochschuldidaktischen Zentrums Sachsen (HDS) und des Arbeitskreises E-Learning der Landesrektorenkonferenz Sachsen (AK E-Learning) mit den staatlichen sächsischen Hochschulen. Es fokussierte schon vor der Covid-19Pandemie die Integration der digitalen Hochschulbildung in die Hochschuldidaktik sowie deren Breitenwirkung. Das Projekt umfasst drei parallele Programmlinien: Digital Fellowships, Digital Workspaces und Digital Change Agents. Das Ziel ist die Unterstützung von sächsischen Hochschulakteur*innen bei der (Weiter-)Entwicklung digital gestützter Lehre, also der Anreicherung, Ergänzung oder Vollvirtualisierung der Lehre durch den Einsatz digitaler Medien mit dem Ziel, „Wissen zu vermitteln, für den zwischenmenschlichen Austausch oder das gemeinsame Arbeiten an digitalen Artefakten. “ (Kerres, 2012, S. 18). ${ }^{3}$

Mit der Programmlinie Digital Fellowships erhalten Hochschullehrende eine finanzielle Unterstützung in Höhe von 12.000€ bzw. 25.000€ für ein Einzel- oder Tandemfellowship, um ihre Lehrveranstaltung unter Nutzung digitaler Werkzeuge inhaltlich und methodisch weiterzuentwickeln.

Bei den Digital Workspaces handelt es sich um hochschuldidaktische Angebote im Werkstattformat, welches es den Teilnehmenden ermöglicht, eigene Schwerpunkte zu setzen und an individuellen Konzepten und Produkten für die eigene (digitale) Lehre zu arbeiten. Die Digital Workspaces werden kollaborativ von den Mitarbeiter*innen der am Projekt beteiligten

\footnotetext{
3 Weitere Informationen zum Projekt und $\mathrm{zu}$ den Programmlinien finden sich unter www.hd-sachsen.de/ web/page.php?id=1176 (16.06.2021).
} 
Hochschulen organisiert und für Lehrende aller staatlichen sächsischen Hochschulen kostenfrei angeboten. Unter den Pandemiebedingungen erfolgte die Durchführung ausschließlich virtuell, zukünftig sind kombinierte oder ausschließliche Präsenzangebote denkbar.

Die Programmlinie Digital Change Agents zielt auf die Entwicklung und Implementierung eines Multiplikator*innenprogramms zur Ausbildung von Personen, die durch die zunehmende Digitalisierung bedingte Change-Prozesse an den sächsischen Hochschulen begleiten. Das Programm richtet sich vorrangig an Lehrende, die an Schnittstellen der Lehrorganisation (z.B. Studiengangs- und Modulverantwortliche) arbeiten und bei der Veränderung von Studium und Lehre aktiv beteiligt sind.

Das Projekt erlangte in der Pandemie besondere Aufmerksamkeit. Es war eng verwoben mit den vorherrschenden Bedingungen, sodass eine gezielte Zusammenarbeit mit den Akteursgruppen möglich war. Dem erhöhten Bedarf an didaktischen Unterstützungsleistungen und Weiterbildungsformaten konnte durch die Angebote der DHS in vielfältiger Weise Rechnung getragen werden.

\section{Methodisches Vorgehen}

Zur Reflexion und Diskussion der Erfahrungen, die während der Ad-hoc-Digitalisierung unter Pandemiebedingungen in Sachsen gesammelt wurden, fanden synchrone Think Tanks mit Akteur*innen der drei Programmlinien des Projektes statt. Unter einem Think Tank verstehen wir in Anlehnung an Poguntke (vgl. 2016) ein Fokusgruppen-Format, in dem sich die interdisziplinär zusammengesetzten Teilnehmenden mit zukunftsgerichteten Fragestellungen beschäftigen, um systematisch Zukunftschancen zu identifizieren. Durch das Auslösen von Kreativität und Dynamik sollen Zukunftsideen entwickelt werden, die es ermöglichen, die Belange der Zielgruppen in künftige strategische Entscheidungsprozesse einfließen zu lassen (vgl. Kühn/Koschel 2018). Die konkrete methodische Ausgestaltung eines Think Tanks ist von der Zielstellung bzw. dem gewählten Thema sowie dem Teilnehmendenkreis abhängig. Grundsätzlich können jedoch analysierende (z. B. mit Blick auf Trends oder sich abzeichnende (technologische) Entwicklungen) und gestaltende Think Tanks (z.B. Entwicklung von Ideen, Konzepten oder Lösungsansätzen) unterschieden werden (vgl. ebd.), bei denen vorrangig Kreativitätstechniken zur Wissenssammlung und -produktion (Brainstorming, Szenario-Technik, Design Thinking etc.; zu Kreativitätstechniken siehe z. B. Backerra/Malorny/Schwarz 2020; Traut-Mattausch/Kerschreiter 2012) in moderierten Gruppen zum Einsatz kommen. Im Rahmen der durchgeführten Veranstaltungen stand neben dem Blick auf die absolvierte Lernkurve im Bereich digitaler Hochschulbildung sowie erzielter (Miss-)Erfolge v. a. die Reflexion darüber im Fokus, was nach der Pandemie an den sächsischen Hochschulen erhalten und worauf auch in Zukunft aufgebaut werden soll. Folglich wurde sowohl analytisch als auch gestaltend gearbeitet und eine Mischform realisiert.

Zu den adressierten Akteursgruppen gehörten 72 Digital Fellows als Repräsentant*innen besonders aktiver Lehrender, 16 Digital Change Agents als Multiplikator*innen des digitalen Wandels im Bereich der Studienorganisation sowie elf Mitarbeitende im Third Space zur Unterstützung der digitalen Hochschulbildung. Insgesamt wurden drei Think Tanks organisiert, die der zuvor benannten Zielsetzung entsprachen. Da die Teilnahme freiwillig erfolgte, wurde 
jeweils mit anfallenden Stichproben (auch: Ad-hoc-Stichprobe; vgl. Döring/Bortz 2016) gearbeitet. Während die Veranstaltungen mit den Digital Fellows und Digital Change Agents parallel stattfanden und einen identischen methodischen Ablauf aufwiesen, wurde der Think Tank mit den Mitarbeitenden im Third Space im Nachgang in verkürzter Form durchgeführt. Dies hatte vor allem organisatorische Gründe, da die Diskussion aufgrund der hohen Arbeitsbelastung der Kolleg*innen im Rahmen der üblichen Prozesse im Kontext des Gesamtprojektes erfolgte, wobei den Kolleg*innen das Konzept der Think Tanks vorab bereits bekannt war und diese sich ohnehin regelmäßig über Erfolge und Misserfolge austauschen.

\section{Ablauf der Think Tanks mit den Digital Fellows und Digital Change Agents}

Nach einem kurzen Einstieg fanden zwei moderierte Runden zum Erfahrungsaustausch, zur Diskussion in Kleingruppen und entlang spezifischer, wählbarer Themen der digitalen Hochschulbildung statt. Die Themen ergaben sich aus einer im Vorfeld durchgeführten Abfrage. ${ }^{4}$ Sie wurden also von den Teilnehmenden selbst eingebracht und unterschieden sich daher in den einzelnen Think Tanks. Die erste Runde konzentrierte sich auf das in Sachsen Erreichte sowie positive und negative Erfahrungen der Teilnehmenden. In der zweiten Runde, welche in den selben Kleingruppen absolviert wurde, standen Ideen und Visionen für die Zukunft im Vordergrund. Diese sollten in Form von Thesen dazu aufbereitet werden, welche Wünsche und Visionen sich aus den Erfahrungen in der pandemiebedingten Online-Lehre ergeben. Die Resultate der Kleingruppenarbeit wurden anschließend im Plenum vorgestellt und anhand einer Punktabfrage durch die Teilnehmenden priorisiert.

\section{Ablaufdes Think Tanks mit den Mitarbeitenden im Third Space}

Im Gegensatz zum zuvor beschriebenen Ablauf verzichtete der Think Tank mit der dritten Akteursgruppe auf den Einstieg, die Abfrage vorab und die erste Runde Kleingruppenarbeit. Stattdessen wurden die von den Digital Fellows und Digital Change Agents bearbeiteten Themen zur Wahl gestellt und die bereits zusammengetragenen Erfahrungen und (Miss-)Erfolge als Arbeitsgrundlage genutzt. Die Teilnehmenden konzentrierten sich also direkt auf Ideen und Visionen für die Zukunft bzw. die Formulierung von Thesen. Der weitere Verlauf des Think Tanks blieb unverändert.

\section{Auswertung und Zusammenfübrung des Erarbeiteten}

Die qualitative Auswertung der Think Tanks erfolgte anhand der Verschriftlichungen, welche die Teilnehmenden im Rahmen der (zwei) Gruppenarbeitsphasen auf digitalen Whiteboards anfertigten. Darüber hinaus wurde die Plenumsphase zur Vorstellung der Ergebnisse der Kleingruppenarbeit aufgezeichnet und zur Rekonstruktion der Argumentationslinien herangezogen. Etwaige vorangegangene mündliche Diskussionsverläufe flossen nicht in die Auswertung ein.

\footnotetext{
${ }^{4}$ Bei der Anmeldung konnte (optional) folgende Frage beantwortet werden: „In Bezug auf welche Aspekte des zwangsweisen digitalen Lehrbetriebs besteht aus Ihrer Sicht der größte Handlungsbedarf? “.
} 
Zunächst wurden die Artefakte aus den Think Tanks für jede Akteursgruppe separat gesichtet und die entstandenen Thesen nach Priorität sortiert, geclustert, ggf. zusammengefasst sowie Formulierungen auf Basis der Dokumentation geschärft. Auf diese Weise entstanden pro Akteursgruppe zwischen 12 und 14 Thesen.

\section{Ergebnisse: Thesen zur Lehre nach Corona}

\section{Thesen der Digital Fellows}

Zum Think Tank der Digital Fellows waren 23 Personen angemeldet. Sie lehren an 10 verschiedenen sächsischen Hochschulen ( 4 Universitäten, 5 Hochschulen für Angewandte Wissenschaften und 1 Kunst-/Musikhochschule). Die im Vorfeld abgefragten und in den Diskussionsgruppen bearbeiteten Themen ließen sich folgenden Bereichen zuordnen: (1) Gestaltung synchroner Veranstaltungen, (2) asynchrones Arbeiten und Betreuung von Studierenden, (3) Umsetzung digitaler Prüfungen.

Unter den Teilnehmenden in der Gruppe Gestaltung synchroner Veranstaltungen war die Aktivität und Aktivierung der Studierenden in synchronen Veranstaltungen ein herausragendes Thema. Sie tauschten sich über verschiedene methodische Umsetzungsmöglichkeiten aus und berichteten von Erfolgen mit dem Flipped-Classroom-Format (zur Methode siehe z.B. Handke/Schäfer 2012; Handke/Sperl 2012), welches nahezu alle Teilnehmenden bereits erprobt hatten. In diesem Kontext verwiesen sie darauf, dass synchrone und asynchrone Lernphasen gleichermaßen strukturiert, geplant und miteinander verknüpft werden und die synchronen Phasen vor allem gemeinsame Aktivitäten, Austausch und Diskussionen unterstützen sollten. Dabei konstatierten sie einen erhöhten Arbeitsaufwand für die Vorbereitung und Begleitung der Szenarien und die Umsetzung aktivierender Methoden. Demzufolge fokussieren die drei von dieser Gruppe entwickelten Thesen genau diese Punkte: Neben der Forderung nach mehr Wertschätzung für das Engagement der Lehrenden bei der Weiterentwicklung ihrer Lehrkonzepte und nach einer weiteren Erprobung von aktivierenden Methoden in synchronen Lehrveranstaltungen erhielt folgende These dieser Gruppe in der anschließenden Priorisierung die meisten Punkte:

„Die Neuausrichtung von Präsenzveranstaltungen kann zusätzlichelandere Lerneffekte erzeugen, daher möchten wir in Zukunft, dass die Wissensvermittlung und Gruppenarbeiten auch komplementär in die asynchrone Lebre ausgelagert werden können und die synchronen Veranstaltungen für Diskussion, Austausch etc. genutzt werden".

In der Kleingruppe zum Thema asynchrones Arbeiten und Betreuung von Studierenden wurde ebenfalls über die Abstimmung von asynchronen und synchronen Lernphasen u.a. mittels des Flipped-Classroom-Konzepts diskutiert und verschiedene Erfahrungen mit digitalen Werkzeugen zur asynchronen Kommunikation vorgestellt. Dabei wurde v.a. betont, dass Gruppenarbeiten im asynchronen Format nützlich erschienen, jedoch eine gesonderte Moderation und Begleitung erforderten, da sich v.a. bei komplexen Aufgabenstellungen teilweise ein Mangel an Selbstorganisationsfähigkeiten seitens der Studierenden offenbarte. Aufgrund der Erfahrungen eines Teilnehmenden mit der Betreuung von asynchronen Gruppenarbeiten durch studentische E-Tutor*innen hielten die Teilnehmenden auch in der Formulierung ihrer fünf 
Thesen die Einbeziehung von Studierenden in die Lehrtätigkeit fest. Diese könnten sowohl in der Lernprozessbegleitung als auch der Weiterentwicklung von Lehrkonzepten eine wichtige Unterstützung für die Lehrenden darstellen. Als weitere Unterstützungsformen nannte diese Gruppe Peer-Formate (z. B. Reverse Mentoring, Co-Creation) in Bezug auf die Erstellung von Lehrmaterialien. Darüber hinaus fordert auch diese Gruppe, dass synchrone und asynchrone Phasen (z. B. über das Flipped-Classoom-Format) zusammengedacht werden sollten und formuliert mit Blick auf die Wertschätzung digital gestützter Lehre eine weitere hochpriorisierte These:

\section{„Digitale Lebre muss begleitet werden und verursacht fortlaufend Aufwand, der deputatsseitig angerechnet werden muss."}

Das Thema Umsetzung digitaler Prüfungen wurde v.a. im Kontext der rechtlichen, technischen und organisatorischen Rahmenbedingungen diskutiert. Technische Hürden und die fehlende Rechtssicherheit insbesondere in Bezug auf Täuschungsversuche standen hierbei im Mittelpunkt. Dennoch erarbeitete die Gruppe gleichermaßen Vorteile und Chancen für das digitale Prüfen, die sie sowohl in der besseren Lesbarkeit der Antworten als auch in der Nachnutzbarkeit von Materialien sahen. Mit ihren fünf Thesen brachte die Gruppe zum Ausdruck, dass digitale Prüfungen beibehalten werden sollten und hierfür entsprechende technische und rechtliche Rahmenbedingungen (weiterhin) zu schaffen seien. Darüber hinaus verwiesen sie auf die lernunterstützende Wirkung von Self-Assessments und die Notwendigkeit der Ausbildung von Medienkompetenz auf Seiten der Studierenden.

\section{Thesen der Digital Change Agents}

Am Think Tank der Digital Change Agents nahmen neun Personen teil. Diese kamen aus sieben verschiedenen sächsischen Hochschulen (3 Universitäten, 3 Hochschulen für Angewandte Wissenschaften, 1 Kunst- und Musikhochschule) und repräsentierten fünf unterschiedliche Statusgruppen (2 Prorektor*innen, 2 Studiendekan*innen, 2 Referent*innen in Stabsstellen der Hochschulleitungen, 2 Mitarbeitende in E-Learning-Serviceeinrichtungen, 1 Lehrkraft für besondere Aufgaben). Ausgehend von der im Vorfeld durchgeführten Abfrage zu Handlungsbedarfen im Bereich digitaler Hochschulbildung wurden die Themen (1) Digitales Prüfen, (2) Gestaltung synchroner Veranstaltungen sowie (3) nachhaltige Weiterentwicklung und Nachnutzbarkeit von im Rahmen der Pandemie entstandenen Konzepte, Inhalten und Materialien in Kleingruppen bearbeitet.

In Bezug auf Digitales Prüfen wurde in der Diskussion deutlich, dass an den Hochschulen noch viele juristische und administrative Fragen ungeklärt seien, die didaktische Fragen häufig überlagerten. Dennoch ließen sich unterschiedliche Formate digitaler Prüfungen gut über die vorhandene Infrastruktur realisieren, da sowohl der Großteil der Lehrenden und Studierenden als auch der Hochschulleitungen viel Flexibilität und Risikobereitschaft mitbrächten. Auch die hohe Belastung aller involvierten Akteur*innen und die Nachteile ausschließlich digitaler Lehr-Lern- und Prüfungsformate wurden thematisiert. Unabhängig davon wurde die Notwendigkeit der hochschuldidaktischen Weiterbildung von Lehrenden im Bereich der Prü- 
fungsdidaktik und der Wunsch nach einem zeitgemäßen Verständnis von Lehre bzw. Prüfungen herausgehoben. Im Ergebnis formulierte die Kleingruppe insgesamt vier Thesen.

Hinsichtlich der Gestaltung synchroner Veranstaltungen herrschte innerhalb der Kleingruppe Einigkeit, dass ausschließlich synchrone Formate keine gute digital gestützte Lehre darstellten, sondern stets um asynchrone Anteile ergänzt werden sollten. Die Frage, was gute digital gestützte Lehre ist, sei jedoch stets individuell zu beantworten, wofür sowohl Möglichkeiten der Vernetzung und Weiterbildung zu schaffen als auch vielfältige Unterstützungsangebote bereitzustellen seien. Als Vorteile synchroner Veranstaltungen wurden die Möglichkeit des direkten Austauschs, der (Klein-)Gruppenarbeit sowie des spielerischen Umgangs mit technischen Hilfsmitteln betont, die didaktisch sinnvoll genutzt werden sollten. Hierbei dürften jedoch die Nachteile nicht aus dem Blick geraten, die sich vor allem aus einer mangelnden technischen Ausstattung (z. B. Netzverfügbarkeit, Hardware) ergäben. Ferner seien die didaktischen Gestaltungsoptionen synchroner Veranstaltungen mit steigender Teilnehmendenzahl zunehmend begrenzt. Die drei formulierten Thesen betonen die Notwendigkeit dauerhafter (personeller) Ressourcen, die Sicherung der technischen Handlungsfähigkeit für die Hochschulen sowie die Bedeutung von Anreizen und eines Rahmens für Austausch und Weiterbildung an den Hochschulen.

Mit Blick auf das Thema nachbaltige Weiterentwicklung und Nachnutzbarkeit wurde zunächst konstatiert, dass die Ad-hoc-Digitalisierung unter Pandemiebedingungen zu einer enormen Erweiterung von Erfahrungen und damit Kompetenzentwicklung aufseiten von Lehrenden und Lernenden geführt hätte - insbesondere was Fragen der Nutzung von Medientechnik angeht. Darüber hinaus sei nicht nur ein Bewusstsein für die Besonderheiten digital gestützter Lehre (Ressourcenbedarf, Gestaltungsmöglichkeiten) sowie die Notwendigkeit der flexiblen Anpassung und Weiterentwicklung der eigenen Lehre entstanden. Auch viele fach-, bereichsund hochschulübergreifende Kooperationen hätten sich etabliert, wodurch ein hochschuldidaktischer Reflexionsprozess über die Qualität von Hochschullehre in Gang gesetzt worden sei. Demgegenüber stünden noch immer erhebliche Unklarheiten in Bezug auf die rechtlichen Rahmenbedingungen (Urheberrecht, Datenschutz, Prüfungsrecht) digital gestützter Lehre. Hinzu käme der Umstand, dass Teile der erarbeiteten Inhalte aufgrund von Limitierungen verschiedenster Art nicht den gewünschten Qualitätsansprüchen genügten (Masse statt Klasse), und die Einsicht, dass weitere (strukturelle) Maßnahmen erforderlich sind, um das durch pandemiebedingte Ad-hoc-Digitalisierung Errungene aufrecht zu erhalten:

„Das Momentum der Ad-hoc-Digitalisierung lässt sich nicht allein durch Mission aufrechterhalten. Vielmebr sind Anreizsysteme und Zielvereinbarungen zu schaffen, die den Einsatz für eine Weiterentwicklung digital gestützter Hochschullebre attraktiv machen. "

\section{Thesen der Mitarbeitenden des Third Space}

Am Think Tank der Mitarbeitenden des Third Space nahmen acht Personen aus acht verschiedenen sächsischen Hochschulen (4 Universitäten, 4 Hochschulen für Angewandte Wissenschaften) teil. In den Kleingruppen wurden die Themen (1) Gestalten synchroner Veranstaltungen, (2) Betreuung asynchroner Kommunikation und (3) digitales Prüfen bearbeitet. 
Hinsichtlich der Gestaltung synchroner Veranstaltungen tauschte sich die Kleingruppe über hybride Formate und die Übertragung von Präsenzformaten in den digitalen Raum aus. Sie konstatierte, wie essenziell dabei die Unterstützung der Lehrenden durch Mitarbeitende des Third Space sei. Dies setze aus ihrer Sicht wiederum voraus, dass auch sie selbst ihre eigenen Medien- und medienpädagogischen Kompetenzen (weiter-)entwickelten, um weiterhin der Zielgruppe entsprechende, qualitativ hochwertige Angebote unterbreiten zu können. Im Zuge der Kleingruppenarbeit entstanden insgesamt drei Thesen, wobei die nachfolgende These die höchste Zustimmung erfuhr:

"In Reaktion auf die spontane Umstellung der Lebre wird eine Vielzabl an Tools und Kommunikationskanälen verwendet, daher möchten wir den Umgang mit diesen optimieren (Umfang, Ökonomie, Datenschutz, Nachbaltigkeit, ...). “

Die Kleingruppe, die sich der Betreuung asynchroner Kommunikation widmete, thematisierte die Bedeutung von Planung und Methodik, aber auch von Moderation. Sie betonte weiterhin die dadurch entstehende Mehrbelastung in der Vor- und Nachbereitung der Angebote sowie die Vielzahl an Herausforderungen, mit denen Lehrende durch die Betreuung asynchroner Angebote insgesamt konfrontiert würden. Daraus resultierten Forderungen nach der Abbildung des erhöhten Aufwands in Deputaten sowie nach Weiterbildungsmöglichkeiten für Lehrende, um den genannten Themen Rechnung tragen, Chancen in den Fokus rücken und gleichzeitig selbst mit gutem Beispiel vorangehen zu können. Die Gruppe hat insgesamt sechs Thesen formuliert.

In der Kleingruppe zum Digitalen Prüfen wurde das Dilemma zwischen dem nötigen Sprung in die Moderne einerseits und der Angst vor der Weitergabe von eigenen Prüfungsaufgaben, die in Umlauf gebracht werden könnten, andererseits aufgezeigt. Die Teilnehmenden stellten fest, dass digitale Prüfungen unter den entsprechenden Voraussetzungen sehr gut umsetzbar seien. In den insgesamt fünf Thesen forderten sie daher auch den künftigen Einsatz von Online-Prüfungen an geeigneten Stellen, die Schaffung dafür notwendiger Voraussetzungen (v.a. mit Blick auf rechtliche Grundlagen, die entsprechende Infrastruktur, finanzielle und personelle Absicherung) sowie die Bereitstellung entsprechender Unterstützungsangebote (Supportstrukturen und Weiterbildungsmöglichkeiten).

\section{Veröffentlichung und Zusammenführung der Thesen}

Im Nachgang der einzelnen Think Tanks wurden die erarbeiteten Thesen von den Projektkoordinatorinnen aufbereitet: Entsprechend der Wichtung durch die jeweilige Gruppe wurden die Formulierungen geschärft, die Thesen geclustert und deren Reihenfolge angepasst. Zunächst wurden die Thesen der Digital Fellows über den Twitterkanal des Bildungsportals Sachsen (Tweets von @eLearningSax) veröffentlicht, im Anschluss die Thesen der Digital Change Agents über Tweets von @HDSachsen verbreitet. Darauf folgten die Thesen der Mitarbeitenden des Third Space, welche abwechselnd auf den beiden Twitterkanälen geteilt wurden. Die bisher veröffentlichten Thesen wurden dabei mehrfach geliked, geteilt und kommentiert. 
Parallel dazu wurde ein gemeinsames Thesenpapier aller drei Akteursgruppen erarbeitet. Dieses wird u.a. über die Internetauftritte des HDS sowie des AK E-Learning veröffentlicht ${ }^{5}$ und an das SMWK kommuniziert. Die entwickelten Thesen werden somit in den öffentlichen Diskurs eingebracht und sollen die Diskussion um die Zukunft der digitalen Hochschulbildung in Sachsen weiter vorantreiben.

\section{Diskussion}

Für die Zukunft der digital gestützten Lehre an sächsischen Hochschulen verbinden die drei befragten Akteursgruppen viele gemeinsame Vorstellungen. So sind sich die Gruppen einig, dass das Thema digitales Prüfen weiterhin vorangetrieben werden soll und die notwendigen technischen, organisatorischen und rechtlichen Rahmenbedingungen hierfür erst noch geschaffen werden sollen. Alle drei Gruppen sind überzeugt, dass durch die digitale Unterstützung synchroner und asynchroner Lernphasen bereits erfolgreiche methodische Umsetzungsformen und digitale Werkzeuge erprobt wurden und hierfür entsprechende Kompetenzen auf Seiten der Lehrenden erworben wurden. Gleichzeitig verweisen alle Gruppen auf die Mebrbelastung (Vorbereitung/Überarbeitung bestehender Konzepte, Materialerstellung, Einarbeitung in Technologien, Betreuung etc.) durch die digital gestützte Lehre und dass diese angerechnet und wertgeschätzt werden sollte.

Hinsichtlich weiterer Unterstützungsformen erarbeiteten die drei Gruppen unterschiedliche Schwerpunkte: Unter den Digital Fellows wurden vor allem Peer-Formate und das Einbeziehen von Studierenden angeregt, wobei die Digital Change Agents und die Mitarbeitenden des Third Space den Stellenwert von Weiterbildungsangeboten deutlich machten. Die Digital Fellows als Gruppe der Hochschullehrenden diskutierten stärker aus einer didaktisch-methodischen Perspektive, während die Digital Change Agents als Vertreter*innen für eine strategische Implementierung von Digitalisierungsprozessen in der Lehre auch Aspekte des Qualitätsmanagements und der Handlungsfreiräume für Lehrende in den Blick nahmen. Die Mitarbeitenden im Third Space verwiesen zusätzlich auf den Unterstützungsbedarf ihrer Statusgruppe und forderten entsprechende Angebote.

Die Ergebnisse der drei Diskussionsrunden geben jeweils statusspezifische Eindrücke aus dem Lehrbetrieb unter Pandemiebedingungen. Sie können aufgrund der anfallenden Stichproben (vgl. Döring/Bortz 2016) nicht als repräsentative Einschätzung für den sächsischen Hochschulraum gelten, sind jedoch geeignet, um ein Stimmungsbild aus unterschiedlichen Perspektiven einzufangen. Aus diesem lässt sich eine Tendenz zu einer Fortführung und stetigen Weiterentwicklung der begonnenen Konzepte und Maßnahmen ablesen, die aufgrund der Spezialisierung des Gesamtprojektes auf die Digitalisierung der sächsischen Hochschullehre wünschenswert und erwartbar ist.

\footnotetext{
${ }^{5}$ Die Thesen sind in Kürze unter www.hd-sachsen.de und bildungsportal.sachsen.de/portal/parentpage/projekte/digital-fellowships/vernetzungstreffen verfügbar.
} 


\section{Fazit und Ausblick}

Insgesamt haben die Think Tanks und das erarbeitete Thesenpapier gezeigt, dass die beteiligten Akteursgruppen in Sachsen ein gemeinsames Verständnis von digital gestützter Lehre nach Corona verbindet. Dabei haben sich Schwerpunkte herauskristallisiert, die als zentral erachtet werden, die Perspektiven der einzelnen Gruppen unterscheiden sich aber durchaus, vor allem was den Blick auf die Voraussetzungen für ein Gelingen digital gestützter Lehre anbelangt. Die insgesamt sehr normative Perspektive auf die Thematik zeigt, dass die Forderungen unverändert sind: Ressourcen, Infrastrukturen und zu klärende rechtliche Rahmenbedingungen werden von allen Beteiligten als essenziell angesehen. Damit wird deutlich, dass sich der Diskurs gegenüber dem vor der Pandemie kaum verändert hat, denn auch Befragungen aus den Jahren 2015 und 2016 kommen zu dem Schluss, dass verlässliche Rahmenbedingungen sowie die eigene Motivation und Medienkompetenz der Hochschullehrenden die förderlichsten Faktoren für den Einsatz digitaler Medien sind (Riedel/Börner 2016; Schulze-Achatz/Riedel 2016). Inwiefern die Pandemiesituation nachhaltige Veränderungen in den Digitalisierungsprozessen an den Hochschulen bewirkt, bleibt daher abzuwarten. Es besteht Hoffnung, dass die pandemiebedingten Einschränkungen die Notwendigkeit einer Digitalisierung von Lehr-/Lernprozessen noch deutlicher machen konnten als die medien- und hochschuldidaktischen Diskurse der vergangenen Zeit und die Forderungen nun mit mehr Nachdruck umgesetzt werden. Darüber hinaus ist nicht nur in den Think Tanks deutlich geworden, dass Digitalisierung nicht nur eine Übertragung analoger Praktiken in den digitalen Raum bedeutet, sondern eine Veränderung des methodischen und didaktischen Handelns bewirken kann. Weitere Digitalisierungsbestrebungen sollen daher weniger die Quantität als vielmehr die Qualität von digital gestützten Lehr- und Lernprozessen in den Blick nehmen. So haben auch die Think Tanks einmal mehr verdeutlicht, dass gute digital gestützte Lehre nicht selbstverständlich ist, sondern engagierter Persönlichkeiten, Reflexion, Unterstützung durch Mitarbeitende des Third Space und vor allem innovativer Ideen bedarf.

Im Zuge der pandemiebedingten Online-Semester ist insgesamt ein stärkeres Bewusstsein für die Besonderheiten digital gestützter Lehre entstanden. Gleichzeitig wurden Kompetenzen für digitale Lehr-Lernszenarien weiterentwickelt, wie die Ergebnisse der Think Tanks zeigen. Nun müssen die Nachhaltigkeit und der Transfer der erzielten Fortschritte in den Regelbetrieb gesichert werden. Hierzu ist neben einer verlässlichen Verstetigung und Weiterentwicklung finanzieller, personeller und technischer Ressourcen (vgl. Junge/Niesyto/Rymeš 2019), die sowohl sachsenweit als auch hochschulspezifisch geplant werden sollten, auch die Erprobung neuer Formate, die Vernetzung der Akteur*innen und die noch stärkere Einbeziehung Studierender in die Lehrveranstaltungsgestaltung fortlaufend mitzudenken. Digitalisierungsprozesse im Bereich der Hochschulbildung haben mit der Pandemie weder begonnen, noch werden sie mit dieser beendet. Die Diskussion um Weiterentwicklungspotenziale muss fortgeführt werden, die gemachten Erfahrungen müssen weiter reflektiert und systematisiert werden, um den digitalen Wandel auch künftig innovativ und sinnvoll gestalten zu können. 


\section{Literatur}

Arbeitskreis E-Learning der Landesrektorenkonferenz Sachsen (AK E-Learning) (in press): Abschlussbericht 2019/2020 des Arbeitskreises E-Learning der Landesrektorenkonferenz Sachsen zur Unterstützung von Vorhaben zur Entwicklung, Stärkung und Verstetigung des E-Learning an sächsischen Hochschulen in den Jahren 2019 und 2020.

Backerra, Hendrik/Malorny, Christian/Schwarz, Wolfgang (2020): Kreativitätstechniken: Kreative Prozesse anstoßen. Innovationen fördern. München: Carl Hanser Verlag.

Döring, Nicola/Bortz, Jürgen (2016): Forschungsmethoden und Evaluation in den Sozial- und Humanwissenschaften. Berlin/Heidelberg: Springer.

Faßbender, Alex/Hasu, Tian/Metzger, Christiane/Szczyrba, Birgit/van den Berk, Ivo (2020): Praxisschock digitales Lernen. Ergebnisse einer Studierendenbefragung zur Online-Lehre in der „CoronaSituation“. In: Das Hochschulwesen, 68 (4+5), S. 109-118.

Frohwieser, Dana/Gaaw, Stephanie/Hartmann, Stephanie/Jablonka, Marcel/Lenz, Karl/Möller, Jonatan/Winter, Johannes (2020): Lehre in der Corona-Pandemie - Überblicksauswertung der zweiten Befragungswelle. Befragung von Lehrenden und Studierenden zur coronabedingten Umstellung auf virtuelle Lehre im Sommersemester 2020. URL: tu-dresden.de/zqa/ressourcen/datei en/publikationen/hochschulmonitoring/Virtuelle_Lehre_2020_Schnellauswertung_zweite_Well e.pdf(15.06.2021).

Handke, Jürgen/Schäfer, Anna Maria (2012): E-Learning, E-Teaching und E-Assessment in der Hochschullehre. Eine Anleitung. München: Oldenbourg.

Handke, Jürgen/Sperl, Alexander (Hrsg.) (2012): Das Inverted Classroom Model. München: Oldenbourg.

Hooffacker, Gabriele (o. J.): „Autokino hinterm Gutenberg-Bau“. Wie die Lehrenden das Sommersemester einschätzen und wie sie sich aufs nächste Semester vorbereiten. Blogbeitrag. URL: idll.ht wk-leipzig.de/was-war-was-kommt/umfragen/umfrage-semesterende-ss2020/ (15.06.2021).

Hooffacker, Gabriele/Gomon, Sebastian (o. J): Mündliche Prüfungen per Videokonferenz kommen gut an. Umfrage des IDLL zu digitalen Prüfungen ergibt große Akzeptanz einzelner Formate. URL: idll.htwk-leipzig.de/was-war-was-kommt/umfragen/umfrage-ende-ws202021/ (15.06.2021).

Junge, Thorsten/Niesyto, Horst/Rymeš, Robert (2019): Überlegungen zur Nachhaltigkeit des Entwicklungsprojekts dileg-SL. In: Junge, Thorsten/Niesyto, Horst (Hrsg.): Digitale Medien in der Grundschullehrerbildung. Erfahrungen aus dem Projekt dileg-SL. Schriftenreihe Medienpädagogik interdisziplinär, Band 12. München: Verlag kopaed, S.319-344.

Karapanos, Marios/Pelz, Robert/Hawlitschek, Patrick/Wollersheim, Heinz-Werner (2021): Hochschullehre im Pandemiebetrieb: Wie Studierende in Sachsen das digitale Sommersemester erlebten. In: MedienPädagogik: Zeitschrift für Theorie und Praxis der Medienbildung, H. 40, S. 1-24. URL: 10.21240/mpaed/40/2021.01.28.X (15.06.2021).

Kerres, Michael (2012): Mediendidaktik: Konzeption und Entwicklung mediengestützter Lernangebote. München: Oldenburg.

Kühn, Thomas/Koschel, Kay-Volker (2018): Gruppendiskussionen. Ein Praxis-Handbuch. Wiesbaden: SpringerVS.

Poguntke, Sven (2016): Corporate Think Tanks. Zukunftsforen, Innovation Center, Design Sprints, Kreativsessions \& Co. Wiesbaden: Springer Gabler. 
Riedel, Jana/Börner, Claudia (2016): Wir tun es, weil es gut ist! Wie Lehrende die Erfolgsfaktoren für den Einsatz digitaler Medien in der Hochschullehre einschätzen. In: Pfau, Wolfgang/Baetge, Caroline/Bendelier, Svenja M./Kramer, Carina/Stöter, Joachim (Hrsg.): Teaching Trends 2016. München/New York: Waxmann, S. 209-220.

Schulze-Achatz, Sylvia/Riedel, Jana (2016): E-Assessment an Hochschulen gemeinsam stärken: Erfahrungen und Erfordernisse. In: HDS.Journal - Perspektiven guter Lehre, Tagungsedition, 2016/1, S. 15-18. URL: nbn-resolving.org/urn:nbn:de:bsz:15-qucosa-202923 (26.07.2021).

Traut-Mattausch, Eva/Kerschreiter, Rudolf (2012): Kreativitätstechniken. In: Angewandte Psychologie für das Projektmanagement. Berlin/Heidelberg: Springer, S. 263-281.

Zawacki-Richter, Olaf (2020): Halb zog sie ihn, halb sank er hin... Covid-19 als Chance für die Digitalisierung von Studium und Lehre? In: Das Hochschulwesen, 68 (4-5), S. 101-108. 


\section{Informationen zu den Autorinnen}

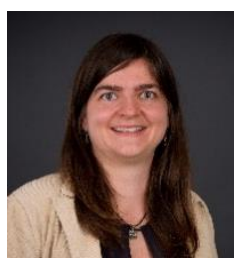

Jana Riedel, M.A. ist Koordinatorin der Programmlinie "Digital Fellowships” im Kooperationsprojekt "Digitalisierung der Hochschulbildung in Sachsen" für den Arbeitskreis E-Learning der LRK Sachsen. Sie bearbeitet seit 2009 verschiedene Forschungsprojekte in den Bereichen digital gestützte Lehr-/Lernszenarien und selbstgesteuertes Lernen an sächsischen Hochschulen.

jana.riedel@tu-dresden.de

Anja Schulz, M.A. ist seit 2008 in verschiedenen Projekten mit Bezug zu digital gestütztem Lehren und Lernen aktiv und aktuell als Koordinatorin der Programmlinie "Digital Change Agents" im Kooperationsprojekt "Digitalisierung der Hochschulbildung in Sachsen” am Hochschuldidaktischen Zentrum Sachsen tätig. Davor arbeitete sie im Verbund der sachsen-anhaltischen Hochschulen am Zentrum für mul-

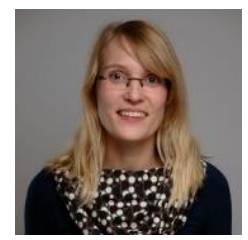
timediales Lehren und Lernen der Universität Halle-Wittenberg, wo sie das Netzwerk „Digitale Hochschullehre in Sachsen-Anhalt" mit initiierte und moderierte.

anja.schulz@hd-sachsen.de

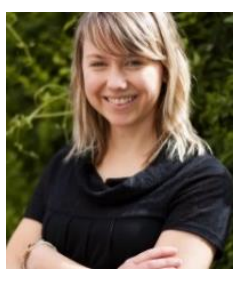

Julia Henschler, M.A. verantwortet als Koordinatorin im Kooperationsprojekt "Digitalisierung der Hochschulbildung in Sachsen” die Programmlinie "Digital Workspaces" am Hochschuldidaktischen Zentrum Sachsen. Sie arbeitet seit 2013 als Hochschuldidaktikerin in Sachsen, u.a. leitete sie das Grundlagenmodul des Sächsischen Hochschuldidaktik-Zertifikats und begleitete Lehrende bei der (Weiter-)Entwicklung innovativer Lehrkonzepte.

julia.henschler@hd-sachsen.de

\section{Zitationshinweis:}

Riedel, Jana/Schulz, Anja/Henschler, Julia (2021): Nach der Ad-hoc-Digitalisierung. Was bleibt? Was soll weg? In: Online-Magazin Ludwigsburger Beiträge zur Medienpädagogik, Ausgabe 21/2021. URL: medienpaed-ludwigsburg.de/ 